\title{
False-Belief Representation and Attribution in Preschoolers: Testing a Graded-Representation hypothesis
}

Nicolas Chevalier and Agnès Blaye

\section{(2) OpenEdition \\ Journals}

Electronic version

URL: http://journals.openedition.org/cpl/233

DOI: $10.4000 / \mathrm{cpl} .233$

ISSN: $1379-6100$

Publisher

Centre PsyCLÉ

\section{Electronic reference}

Nicolas Chevalier and Agnès Blaye, «False-Belief Representation and Attribution in

Preschoolers: Testing a Graded-Representation hypothesis », Current psychology letters [Online], 18,

Vol. 1, 2006 | 2006, Online since 26 April 2006, connection on 08 September 2020. URL : http:// journals.openedition.org/cpl/233; DOI : https://doi.org/10.4000/cpl.233

This text was automatically generated on 8 September 2020

(C) All rights reserved 


\title{
False-Belief Representation and Attribution in Preschoolers: Testing a Graded-Representation hypothesis
}

\author{
Nicolas Chevalier and Agnès Blaye
}

One of the most important milestones in preschool development is the formation of a theory of mind (ToM) at about 4 years of age. ToM encompasses both the understanding that people may have different mental representations of the world that guide their behaviors, and the attribution of mental states (such as beliefs, intentions, or desires) to others and self. A widely used measure designed to tap this cognitive acquisition is the false-belief task (Wimmer \& Perner, 1983). In this task, children see a character put an object in one of two locations. While the character is away, the object is moved to the second location. The children are then asked to predict in which location the character will look for the object. Generally, 3-year-old children fail to attribute a false belief to the character and predict his actions accordingly, while the majority of 4-year-olds succeed (for a review, see Wellman, Cross, \& Watson, 2001).

Evidence has recently accumulated in favor of a close relationship between ToM and executive functions, whose developmental patterns are parallel. "Executive functions" is an umbrella term referring to the "cognitive processes that underlie flexible goaldirected behavior" (Hughes, 2002, p. 69). Executive measures are positively correlated with ToM tasks (Carlson \& Moses, 2001). In one of these measures, the Dimensional Change Card Sort task (DCCS; Frye, Zelazo, \& Palfai, 1995), children are instructed to sort cards according to a first pair of rules (e.g., color rules), and then to switch to a second pair of rules (e.g., shape rules). The DCCS and the false-belief task display the same developmental pattern and are positively correlated (e.g., Frye et al., 1995). Moreover, 3-year-olds show some rigidity on the prepotent response in both tasks. In the DCCS, they perseverate even though the new rules are repeated before each trial. Similarly, they answer on the basis of reality even when they are explicitly told about the character's false belief (Wellman \& Bartsch, 1988). Finally, Kloo and Perner (2003) trained children on either the false-belief task or the DCCS. The results revealed mutual 
transfer effects between the two tasks, providing further evidence for a close relationship between ToM and executive functions.

Several theoretical proposals have been made in an attempt to account for this relationship. For instance, according to the Cognitive Complexity and Control (CCC) theory (Zelazo et al., 1995; Zelazo, Müller, Frye, \& Marcovitch, 2003), the DCCS and false-belief tasks are characterized by the same hierarchical rule structure. In both tasks, children must switch between two setting conditions in order to determine how to respond: they must switch between color and shape rules in the DCCS, and between the character's perspective and reality in the false-belief task. Perspective-switching is possible once children have established a higher-order rule that guides them in deciding when to reason from each perspective. Such higher-order rules are beyond the scope of the cognitive complexity that children can manage before the age of 4 or 5 , which explains why younger children fail in both the DCCS and false-belief tasks.

CCC theory implicitly postulates that inhibitory processes (allegedly required by both tasks) are subordinate to the ability to establish a higher-order rule (Happaney \& Zelazo, 2003). By contrast, a more direct role is ascribed to inhibitory control in Leslie's modular theory (e.g., Scholl \& Leslie, 2001). According to the latter theory, mental-state understanding depends on two modules. The first, the Theory-of-Mind Mechanism (TOMM), is devoted to processing behaviors and the underlying mental states. However, TOMM is assumed to automatically attribute beliefs that match reality. In false-belief situations, TOMM's initial response must be inhibited by Selection Processing (SP, a domain-general inhibitory mechanism). Leslie's theory thus hypothesizes that 3-year-olds fail in the false-belief task because of an immature SP, i.e., immature inhibitory control.

Although it is often acknowledged that inhibitory control is one of the main executive components of the false-belief task, other executive functions may be involved. According to traditional inhibition + working memory accounts, the false-belief task requires the ability to inhibit a prepotent response and to switch between perspectives that have to be held in working memory. Thus, both inhibitory control and working memory need to be sufficiently developed to enable correct false-belief responses. In support of this account, false-belief performance has been found to be more highly correlated with executive measures involving both inhibition and working memory than measures of inhibition only (Carlson, Moses, \& Breton, 2002; Hala, Hug, \& Henderson, 2003).

In line with inhibition + working memory accounts, the graded-representation framework proposed by Munakata (2001) acknowledges the role of both memory and inhibition, but goes further by suggesting how they are interrelated. This model hypothesizes that representations are graded in nature, with the strength of a representation depending on the amount of environmental support for it, and on the individual's developmental state and neurological integrity. Furthermore, there are two types of representations (or memory traces): active and latent (Morton \& Munakata, 2002). Latent representations rapidly develop in posterior cortical areas when a stimulus is processed, and influence stimuli processing later on (a latent bias emerges). In contrast, active representations rely on the number of recurring connections in the prefrontal cortex (they increase during the preschool years). An active representation is formed whenever a stimulus representation is actively maintained. The two types of representations may compete in a task and the amount of 
conflict is dependent on their respective strengths. A given task may only require a weak representation to be correctly performed (if no latent representations compete) while another task may tap the same representation and be incorrectly performed because more strength is needed (to overcome a latent representation). Therefore, inhibitory control depends on the relative strengths of the representations simultaneously involved, which in turn vary as a function of the child's memory resources.

The purpose of this study was to assess the validity of the graded-representation framework in the field of ToM. In this approach, two conflicting representations may compete in the false-belief task: the representation of the character's perspective and that of the perspective of reality. From past experiences, children may have acquired a latent bias to infer beliefs in reference to what they know of reality because in most situations beliefs are true. Therefore, the perspective of reality may lead to a very strong representation. As recurring connections are poorly developed, 3- to 4-year-olds may only build a weak representation of the character's perspective. This discrepancy may lead them to incorrectly base their responses on the perspective of reality. In contrast, older children's development of recurring connections may enable them to maintain a strong active representation of the character's perspective and thus successfully perform the task. Previous findings seem to support this hypothesis. First, when there is no conflict between the latent representation and the representation of reality (i.e., children are given no information about the new location), 3-year-olds correctly base their responses on the character's perspective (Wellman \& Bartsch, 1988). Second, as shown by Clements and Perner (1994) who videotaped children's looking behavior while they performed a false-belief task, children as young as 2;11 correctly glanced at the original location when questioned about where the character would look for the object but did not correctly predict the character's actions before the age of 4 years. Younger children's correct looking in anticipation suggests that their representation of the character's perspective is strong enough for this particular measure but is not for a more traditional pointing/naming criterion. Thus, the gradedrepresentation model accounts for inhibition and memory demands by emphasizing the discrepancy between a strong representation of the reality perspective and a weak representation of the character's perspective.

The graded-representation framework suggests that even in a conflicting situation (in which children have information about the object's new location), reducing the strength difference between the two representations should favor correct responding on the basis of the character's perspective, and thereby improve performance. To test this hypothesis, we created two new versions of the false-belief task, both aimed at reducing this strength discrepancy. In the "two-characters" version, a new character whose perspective matched that of reality was introduced in order to confer symmetric statuses to the perspectives of the critical character and reality. This manipulation should help children realize that many perspectives can be involved in this task, and by prompting the comparison of information available to the two characters, it should help them switch from the perspective of reality to the critical character's perspective ${ }^{1}$.

To our knowledge, attributing symmetric statuses to the representations simultaneously involved in a task has only been attempted for the appearance-reality task, in which children are questioned about a deceiving object whose function and appearance are incongruent (e.g., a sponge that looks like a rock). Plumet and Melot (2002) used a modified version in which they projected hand shadows that looked like 
animals on a wall. Children were placed so that they could simultaneously see the experimenter's hands and their animal-like shadows while answering the traditional reality and appearance questions. The results showed that making both representations of the deceiving object physically present throughout the task improved 3-year-olds' performance. Plumet and Melot's interpretation is in line with the graded-representation model: physically dissociating representations made them equally salient, and this facilitated activation/inhibition processes. In the false-belief task, two earlier studies (Robinson \& Mitchell, 1995; Perner, Lang, \& Kloo, 2002) have already used a second character, but only in justification versions of the task where the children were asked to explain why a character looked for the object in the first location.

Another specificity of the present study consisted in asking children three new questions before the traditional test question. The children had to say where each character was while the object was being moved, whether each of them saw the move, and if they knew where the object was at the present time. These new questions about each character were expected to strengthen the balance between the two characters' perspectives, and thus to help children contrast them and get a better grasp of the existence of two representations about the same reality. Finally, in the "twocharacters+stickers" version, the introduction of the additional character was accompanied by the presence of stickers used as memory cues to decrease demands on working memory (Carlson et al., 2002; Hala et al., 2003). The children were asked to put a sticker on each character. The color of the sticker had to match the color of the cupboard in which the character last saw the chocolate. By substituting for memory resources, the stickers should allow children to maintain a stronger active representation of the critical character's perspective, and should thereby reduce the discrepancy between it and the reality perspective.

Traditionally, success and failure on the false-belief task have been considered as evidence for children's knowledge or lack of knowledge about mental states, respectively. However, the standard version may mask intermediate levels of understanding. This task involves at least two qualitatively different conceptual aspects: it requires children (a) to understand that different representations of a given reality coexist, and (b) to be able to attribute the right representation to each of them. The ability to attribute different representations about the same object or event, but doing so incorrectly, may be indicative of the first conceptual aspect. Thus, introducing a second character into the task might reveal this type of intermediate knowledge.

In summary, the present study was aimed at testing the validity of a gradedrepresentation account of preschoolers' performance on the false-belief task. Two new versions of the task were designed. In the "two-characters" version, the introduction of an additional character should reduce the latent bias in favor of the reality perspective by attributing more symmetric statuses to the two representations involved in the task; this should help children understand that the characters have different representations of the object's location (first conceptual aspect). The gradedrepresentation account predicts that children will perform better on this version than on the standard one. Because the stickers are new environmental cues that reinforce the character's perspective which preschoolers tend to ignore, the "twocharacters+stickers" version should be even easier than the other two versions. As the stickers are also cues promoting the coexistence of two representations and indicative of which representation corresponds to which character, they may help children make 
correct attributions for each character (second conceptual aspect). Finally, although the main purpose of this study was to test the graded-representation model, our predictions can be compared to predictions derived from alternative executive accounts. Given that the additional character and the stickers do not alter how the task is hierarchically structured since children still have to switch perspectives, CCC theory suggests that there will be no differences across the three versions. By contrast, TOMM/SP theory emphasizes inhibition demands in the standard false-belief task, and therefore predicts that introducing a second character should be helpful (giving the same format to the two perspectives should make the reality perspective easier to inhibit) and the stickers should not generate an additional benefit.

MethodParticipants

One hundred and fourteen children (58 girls and 56 boys) were recruited from two French preschools. They ranged in age from 2;11 to 4;11 (mean age: 46.8 months; standard deviation: 6.6 months). The children were split into two age groups: sixty 3year-olds (from $2 ; 11$ to $3 ; 11, \mathrm{M}=41.4$ months; $\mathrm{SD}=2.9$ months) with 20 children per condition, and fifty-four 4-year-olds (from 4;0 to $4 ; 11 ; \mathrm{M}=53$ months; $\mathrm{SD}=3.3$ months) with 18 children per condition.

Materials

The procedure was adapted from Wimmer and Perner (1983). Participants sat in front of a $60 \times 30 \times 25 \mathrm{~cm}$ puppet theater on which two match boxes, one green and one blue, representing cupboards were hung. Two child puppets and one adult puppet (differing in size, hairdo, and clothes) played the roles of Thomas, Julie, and their mother. Only Thomas and the mother were used in the standard version. All three puppets appeared in the "two-characters" and "two-characters+stickers" versions. The mother moved the object (chocolate) from the green to the blue location. Thomas was absent during the move and Julie was present. The children's understanding of the story was assessed by two control questions:

Finally, the children were asked to predict each character's action:

In the "two-characters+stickers" version, the children were asked to select green or blue stickers corresponding to the green and blue cupboards and to stick one on each character to help remember the location of the chocolate. A sticker had to be attributed to Thomas just before he left the scene. A second sticker had to be attributed to Julie before Thomas's return. None of the participants chose the wrong stickers. The children's responses (naming/pointing) to each question were recorded.

Procedure

The participants were tested individually in a quiet room of their preschool. Before the task started, we made sure they could discriminate between the two cupboard colors, and between the different characters (whose differentiating characteristics were highlighted). Each child performed one of the three versions of the false-belief task [standard, "two-characters" (2C), "two-characters+stickers" (2C+S)].

Results

All participants answered the control questions correctly (Memory and Reality) which shows that they remembered where the object was placed first and where it was moved. A few participants hesitated or did not answer. Following Clements and Perner's (1994) procedure, the story was repeated once (for two 3-year-olds in the $2 \mathrm{C}$ condition, one 3-year-old and one 4-year-old in the standard condition) or twice (one 3- 
year-old in the standard condition, and another 3-year-old in the $2 \mathrm{C}+\mathrm{S}$ condition) until they gave correct answers.

In the $2 \mathrm{C}$ and $2 \mathrm{C}+\mathrm{S}$ conditions, the questions were asked about each character whereas in the standard condition the questions only pertained to the critical character. Consequently, the results will be presented in two steps. First, we will analyze the responses about the critical character only so that scores across conditions can be compared. Second, we will see what considering responses about both characters reveals, although all questions about the additional character led to very high success rates (between $75 \%$ and $100 \%$ ).

Before the test question, participants had to answer a series of new questions (Place, See, and Know). The meaning of the Know question ("Does Thomas/Julie know where the chocolate is now?") appeared ambiguous to the children since it could be understood as "Does Thomas/Julie know that the chocolate's location has changed?" (as we intended) with correct answers being "no" for Thomas and "yes" for Julie, or "Has Thomas/Julie got an idea of where the chocolate is?" with correct answers being "yes" for both characters. We therefore discarded this question from the analysis ${ }^{2}$. Success rates for Place were very high for both age groups and across all conditions and ranged from $85 \%$ to $100 \%$ (Table 1), which suggests, in conjunction with the control questions, that the story was well understood. In contrast, success rates for the See question improved, rising from $20 \%$ at age 3 to $74 \%$ at age $4\left[x^{2}(1, N=114)=19.2, p<\right.$. 0001]. Performance for See was quite high for 4 -year-olds in all conditions $(72 \%$ in standard, and $89 \%$ in both $2 \mathrm{C}$ and $2 \mathrm{C}+\mathrm{S}$ ). By contrast, the 3-year-olds' performance on this question increased across conditions from $30 \%$ correct in the standard condition to $40 \%$ in $2 \mathrm{C}$ and $65 \%$ in $2 \mathrm{C}+\mathrm{S}$, with 3-year-olds in $2 \mathrm{C}+\mathrm{S}$ significantly outscoring 3-year-olds in the standard condition $\left[\chi^{2}(1, N=20)=4.91, p=.027\right]$. Moreover this question was positively correlated with the test question $(r=.45, p<.0001)$. The results for the new questions suggest that children displayed a relatively consistent pattern of responding across questions.

Next, performance on the test question for the critical character was analyzed (success rates are given in Table 1). Chi-square analyses revealed a highly significant effect of age, with $28 \%$ success at 3 years and $74 \%$ at 4 years $\left[x^{2}(1, N=114)=23.8, p<.0001\right]$. The age effect occurred within each separate condition $\left[\chi^{2}(1, N=38)=6.9, p=.008\right.$ for the standard condition, $\chi^{2}(1, N=38)=8.7, p=.003$ for the $2 C$ condition, and $\chi^{2}(1, N=38)=9.7$, $p=.002$ for the $2 \mathrm{C}+\mathrm{S}$ condition]. As expected, success rates increased significantly across conditions [34\% of success in the standard condition, $53 \%$ in $2 \mathrm{C}$, and $63 \%$ in $2 \mathrm{C}+\mathrm{S} ; \chi^{2}(2$, $N=114)=6.53, p=.038]$. Pairwise comparisons showed that $2 \mathrm{C}$ children tended to outperform standard condition children $\left[\chi^{2}(1, N=76)=2.62, p=.10\right]$. This tendency is worth mentioning, since the chi-square test was used here in a traditional bilateral analysis, but as suggested by Howell (1997), this significance level can be divided by two because of our specific prediction that children in $2 \mathrm{C}$ would outperform those in the standard condition. The $2 \mathrm{C}+\mathrm{S}$ version led to significantly higher performance than the standard version $\left[\chi^{2}(1, N=76)=6.4, p=.012\right]$. Taken separately, this improvement was significant at age $4\left[\chi^{2}(1, N=36)=5, p=.026\right]$ whereas it only tended to be so at age $3\left[\chi^{2}\right.$ $(1, N=40)=3.1, p=.076]$. In summary, as predicted by the graded-representation framework, the introduction of an additional character tended to help children attribute a false belief to the critical character, and this beneficial effect was greater when the stickers were also introduced. 
Table 1: Success rate for the Place, See, and Test questions about the critical character (absent while the object was being moved).

\begin{tabular}{ccccc}
\hline \multirow{2}{*}{ Question } & Age & \multicolumn{3}{c}{ Condition } \\
\cline { 3 - 5 } & & Standard & $2 \mathrm{C}$ & $2 \mathrm{C}+\mathrm{S}$ \\
\hline \multirow{2}{*}{ Place } & 3-year-olds & $85 \%$ & $85 \%$ & $95 \%$ \\
& 4-yea-olds & $94 \%$ & $100 \%$ & $100 \%$ \\
& Together & $89 \%$ & $92 \%$ & $97 \%$ \\
\hline \multirow{2}{*}{ Sce } & 3-ycar-olds & $30 \%$ & $40 \%$ & $65 \%$ \\
& 4-year-olds & $72 \%$ & $89 \%$ & $89 \%$ \\
& Together & $50 \%$ & $63 \%$ & $76 \%$ \\
\hline \multirow{2}{*}{ Test } & 3-year-olds & $15 \%$ & $30 \%$ & $40 \%$ \\
& 4-year-olds & $56 \%$ & $78 \%$ & $89 \%$ \\
& Together & $34 \%$ & $53 \%$ & $63 \%$ \\
\hline
\end{tabular}

Turning now to the children's performance regarding the additional character, few participants answered incorrectly on the test question concerning this character, whose perspective matched that of reality. These errors concerned only 10 out of 76 children (five 3-year-olds and three 4-year-olds in $2 \mathrm{C}$, and two 3-year-olds in $2 \mathrm{C}+\mathrm{S}$ ). Eight of these 10 participants reversed the two characters' beliefs, predicting that the character who was absent during the move would look for the object in the new location, while the character who remained present throughout the story would look for it in the original location. As suggested in the introduction, this error pattern may reveal an intermediate level of understanding, namely, the existence of two different beliefs about the same reality. New analyses were conducted for the proportion of responses revealing acknowledgment that a false belief could be involved in the situation, irrespective of whether it was attributed to the right character. A significant difference was found between the standard and $2 \mathrm{C}$ conditions for all participants together $\left[\chi^{2}(1, N=76)=8.9, p=.002\right]$, and within each age group $\left[\chi^{2}(1, N=20)=5.6, p=.018\right.$ for 3-year-olds, $\chi^{2}(1, N=18)=5, p=.026$ for 4-year-olds]. Thus, the results suggest that both two-character versions helped the children realize that two different beliefs about the object's location could coexist. Moreover, the rates of false-belief acknowledgment (attributing a false belief to any character) were identical in the $2 \mathrm{C}$ and $2 \mathrm{C}+\mathrm{S}$ conditions, with $50 \%$ of the 3 -year-olds ( 10 children per condition) and $89 \%$ of the 4 -year-olds ( 16 children per condition) being able to attribute a false belief (Figure 1). This lack of a difference between $2 \mathrm{C}$ and $2 \mathrm{C}+\mathrm{S}$ when inversions were taken into account, and the quasi-absence of inversions in $2 \mathrm{C}+\mathrm{S}$, suggest that the stickers helped the children make correct belief attributions to each character.

Figure 1. False-belief acknowledgment (in \%) with or without correct attributions (by age group). 


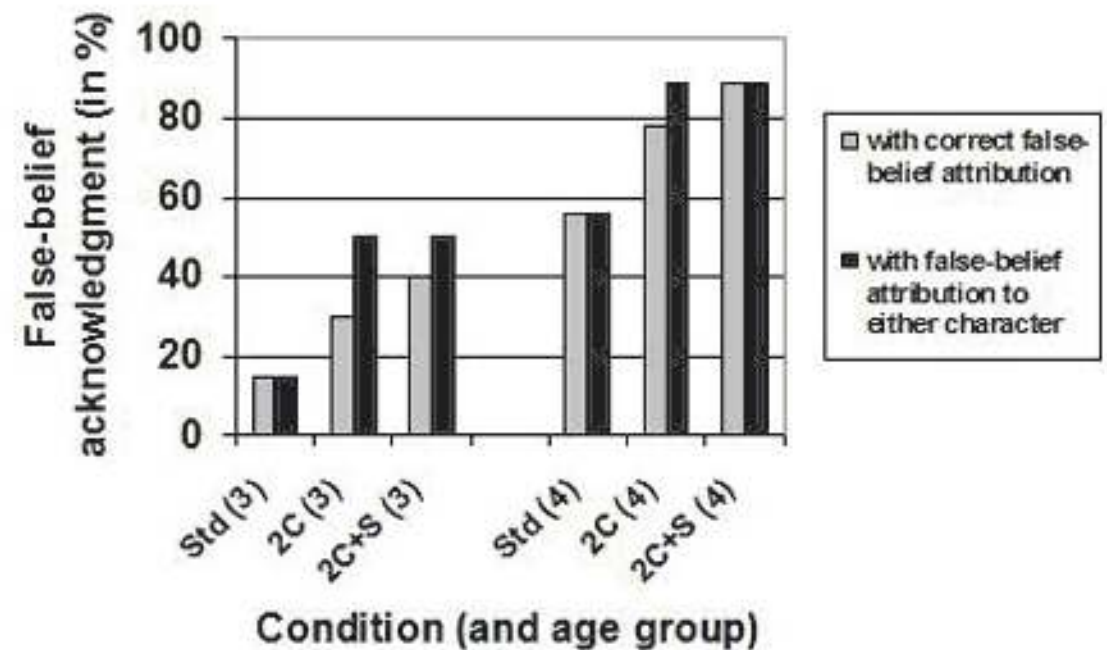

Discussion

In the present study, two new versions of the false-belief task were designed to test a hypothesis derived from the graded-representation model (Munakata, 2001). In line with this hypothesis, performance improved as the difference in strength between the representations of the critical character's perspective and the perspective of reality decreased (standard, "two-characters", and "two-characters+stickers" versions), although the difference was marginally significant for the "two-characters" version. Moreover, the new versions revealed a potential intermediate-level of understanding in which two different beliefs about the same reality are represented but incorrectly attributed to their respective holders.

An additional character that materialized the perspective of reality was introduced to give it a status that was more directly comparable to the critical character's perspective. It was intended to limit children's bias of attributing beliefs from what they know of reality. The idea was to help them realize that several beliefs coexist in this task, and lead them to reason on the basis of the informational state of each character. The superiority of the "two-characters" version over the standard one was marginally significant, which suggests that the introduction of the second character may not have been sufficient to make both perspectives equally salient. Having an additional character was more beneficial when it was accompanied by stickers as in the "two-characters+stickers" version, which led to significantly better performance than in the standard task. The stickers may be seen as environmental cues that substitute for recurring connections and thus allow for a stronger representation of the critical character's perspective. The success gradation pattern found here across the standard version (characterized by an important strength difference that was detrimental to the critical character's perspective), the "two-characters" version (which reduced this difference by weakening the representation of the reality perspective), and the "twocharacters+stickers" version (which both weakened the reality perspective and strengthened the critical character's perspective) is consistent with the gradedrepresentation model. 
Alternative executive approaches to false-belief performance have been advanced. CCC theory (Frye et al., 1995) hypothesizes that young children fail this task because its hierarchical rule structure exceeds the cognitive complexity level they can manage. However, the additional character and stickers used in our modified versions did not change how the false-belief task was hierarchically structured, since the children still had to switch between two perspectives. Thus, the present results do not support CCC theory. By contrast, the beneficial effect of introducing the second character, which can be interpreted as reducing inhibitory demands, is in line with executive accounts that emphasize inhibitory demands in ToM tasks (such as TOMM/SP theory; e.g., Scholl \& Leslie, 2001), which, however, do not provide a satisfactory explanation for the additional effect of the stickers. Moreover, note that it can only be inferred from inhibition accounts that inhibition depends on the degree of salience. Munakata's (2001) graded-representation model is the only account that explicitly focuses on salience differences across the representations involved in a task, and that states how memory resources and inhibition are related to these differences. The latter account is thus the only one that directly predicts how performance would evolve across the standard and modified versions of the false-belief task.

Although our results highlight the role of executive aspects in the false-belief task, they do not deny the role of conceptual development. On the contrary, they reveal a potential intermediate-level of knowledge. Both two-character versions enabled significantly more participants to consider the coexistence of two distinct beliefs about the same reality. However, considering distinct beliefs was not always accompanied by correct attributions. This suggests that children's knowledge about false beliefs is a graded rather than all-or-none phenomenon, and accepting that different beliefs coexist without correctly attributing them may constitute an intermediate level of understanding. As most of the reversed attributions occurred in the "two-characters" version, it seems plausible that the additional character helped the children see that each perspective gave rise to a different belief, but the beliefs were reversed because of working memory saturation, since inversions occurred less often when more memory cues were provided (stickers). Further research is needed to clarify this issue.

Furthermore, before predicting the characters' actions, children were asked whether the critical character saw the move (See). Three-year-old performance on this question improved across conditions and was correlated with Test-question performance. The condition effect suggests that in the standard task a very salient representation of the reality perspective causes difficulty in inferring what one sees from one's situational conditions, whereas children as young as 18 months successfully infer what one sees and knows from one's situational conditions in situations involving no false beliefs (e.g., Olineck \& Poulin-Dubois, in press). Making the perspective of reality less salient thus decreases this difficulty. The correlation between the See and Test questions suggests that success on the See question might be a potential mediator of false-belief understanding.

In an important meta-analysis, Wellman et al. (2001) pointed out two limitations of attempts to reduce the executive load of the false-belief task, both of which apply to the present study. First, these authors interpret the fact that young children never perform above the chance level as indicative of a lack of false-belief understanding. By contrast, the graded-representation model hypothesizes that 3-year-olds do have some knowledge about false beliefs but this knowledge is overridden by what they know of 
reality. The performance pattern on the See question and its correlation with the Test question suggest that the modified versions in the present study truly helped children answer on the basis of the critical character's perspective. Second, according to Wellman et al. (2001), if 3-year-olds fail the task because of executive deficits that 4year-olds do not have, then reducing executive demands should not alter 4-year-olds' performance. Yet 4-year-olds also benefit from such reductions. This point is relevant only if there is a ceiling effect in 4 -year-old performance. In the present study, only $56 \%$ of the 4-year-olds successfully passed the standard task, which is comparable to many previous studies showing that a large proportion of children still answer erroneously at 4 (e.g., Wimmer \& Perner, 1983; Siegal \& Beatie, 1991; Robinson \& Mitchell, 1995). An interesting study by Nadel and Melot (2001) showed that 4- to 6year-olds' performance is still influenced by the assessment modality, thus suggesting that reasoning about mental states continues to progress after 4 years. Accordingly, the lack of ceiling performance is consistent with findings indicating that many 4-year-olds are likely to benefit from a reduction of the salience discrepancy between the perspectives involved in the task.

The present study suggests that reducing the representational-strength difference between the reality perspective and the false belief perspective can allow 3- and 4-yearolds to break away from their own prepotent representation of reality, and hence consider the false-belief point of view. As such, this study adds further evidence in favor of the graded development of an understanding of the mind.

\section{BIBLIOGRAPHY}

Carlson, S. M., \& Moses, L. J. (2001). Individual differences in inhibitory control and children's theory of mind. Child Development, 72, 1032-1053.

Carlson, S. M., Moses, L. J., \& Breton, C. (2002). How specific is the relation between executive function and theory of mind? Contributions of inhibitory control and working memory. Infant and Child Development, 11, 73-92.

Clements, W. A., \& Perner, J. (1994). Implicit understanding of belief. Cognitive Development, 9 , 377-395.

Frye, D., Zelazo, P. D., \& Palfai, T. (1995). Theory of mind and rule-based reasoning. Cognitive Development, 10, 483-527.

Hala, S., Hug, S., \& Henderson, A. (2003). Executive functions and false belief understanding in preschool children: Two tasks are harder than one. Journal of Cognition and Development, 4, 275-298.

Happaney, K., \& Zelazo, P. D. (2003). Inhibition as a problem in the psychology of behaviour. Developmental Science, 6, 468-470.

Howell, D.C. (1997). Statistical Methods for Psychology. Pacific Grove, CA : Duxbury/Thomson Learning 
Hughes, C. (2002). Executive functions and development: Why the interest? Infant and Child Development, 11, 69-71.

Kloo, D., \& Perner, J. (2003). Training transfer between card sorting and false belief understanding: Helping children apply conflicting descriptions. Child Development, 74, 1823-1839.

Morton, J. B., \& Munakata, Y. (2002). Active versus latent representations: A neural network model of perseveration, dissociation, and decalage. Developmental Psychobiology, 40, 255-265.

Munakata, Y. (2001). Graded representations in behavioral dissociations. Trends in Cognitive Sciences, 5, 309-315.

Nadel, J., \& Melot, A.-M. (2001). How clear is the 4-year-olds' "clear-cut change" in understanding mind? Cognitive Development, 15, 153-168.

Olineck, K. M., \& Poulin-Dubois, D. (in press). Infants' ability to distinguish between intentional and accidental actions and its relation to internal state language. Infancy.

Perner, J., Lang, B., \& Kloo, D. (2002). Theory of mind and self-control: More than a common problem of inhibition. Child Development, 73, 752-767.

Plumet, M.-H., \& Melot, A.-M. (2002). Helping young children to distinguish appearance from reality: The role of consecutive versus simultaneous dissociation of representations. Current Psychology Letters, 9, 7-21.

Robinson, E. J., \& Mitchell, P. (1995). Masking of children's early understanding of the representational minds: Backwards explanation versus prediction. Child Development, 66, 1022-1039.

Siegal, M., \& Beattie, K. (1991). Where to look first for children's knowledge of false beliefs. Cognition, 38, 1-12.

Scholl, B. J., \& Leslie, A. M. (2001). Minds, modules, and meta-analysis. Child Development, 72, 696-701.

Wellman, H. M., \& Bartsch, K. (1988). Young children's reasoning about beliefs. Cognition, 30, 239-277.

Wellman, H. M., Cross, D., \& Watson, J. (2001). Meta-analysis of theory of mind development: The truth about false belief. Child Development, 72, 655-684.

Wimmer, H., \& Perner, J. (1983). Beliefs about beliefs: Representation and constraining function of wrong beliefs in young children's understanding of deception. Cognition, 13, 103-128.

Zelazo, P. D., Müller, U., Frye, D., \& Marcovitch, S. (2003). The development of executive function in early childhood. Monographs of the Society for Research in Child Development, 68 (3, Serial number 274).

\section{NOTES}

1. As interestingly suggested by an anonymous reviewer, it may be argued that materializing children's own perspective makes it even more salient and difficult to inhibit Yet based on the idea of graded representations, materializing the two perspectives via the same medium (two puppets) and asking children about both characters should prompt them to compare the two characters, and focus on their differences. We believe that in producing a shift of attention from the perspective of 
reality to the contrast that might exist between the two characters' perspectives, the "two-characters" version provides a context for two equally salient representations and hence reduces the prepotency of the reality perspective.

2. No differences across conditions were observed for the Know question ( $45 \%$ success in standard and $2 \mathrm{C}+\mathrm{S}, 47 \%$ in $2 \mathrm{C}$ ).

\section{ABSTRACTS}

Preschoolers'difficulty in the false-belief task has generally been attributed to a deficit in falsebelief representations. However, the standard version of the task does not allow one to distinguish between the ability to represent and the ability to attribute false beliefs. In order to disentangle these two abilities, 114 3- and 4-year-olds were tested on one of two modified versions of the task or on the standard version. In the first modified version, an additional character whose perspective matched that of the participants was introduced in order to give similar statuses to the two perspectives at stake; this was designed to help children understand that different beliefs about a given reality can coexist. In a second modified version, the introduction of the second character was accompanied by stickers used as memory cues for correct belief attributions. As expected, the modified versions turned out to be easier than the standard one. Moreover, some children attributed both true and false beliefs but reversed them, which may reflect an intermediate level of knowledge. The results are discussed in terms of Munakata's (2001) graded-representation model.

On attribue généralement les difficultés des jeunes enfants à la tâche de la fausse croyance à un défaut de représentation. Cependant, la version standard de l'épreuve ne permet pas de distinguer les capacités de représentation et d'attribution d'une fausse croyance. Afin de mieux apprécier chacune de ces capacités, on a proposé à 114 enfants de 3 et 4 ans deux nouvelles versions ou la version standard de l'épreuve. Dans une première variante, on a introduit un second personnage dont le point de vue correspondait à celui des participants afin de conférer des statuts similaires aux deux points de vue en jeux dans la tâche, et ainsi d'aider à la compréhension que différentes croyances à propos d'une même réalité peuvent co-exister. Dans une seconde variante, l'introduction du second personnage était accompagnée d'étiquettes afin de favoriser l'attribution correcte des croyances. Ces nouvelles versions ont effectivement été mieux réussies que la standard. En outre, certains enfants ont attribué une vraie et une fausse croyances mais ont inversé les personnages, laissant apparaître un éventuel niveau intermédiaire de connaissance. Les résultats sont discutés en référence au modèle des représentations graduées de Munakata (2001).

\section{INDEX}

Keywords: executive functions, false belief, graded representations, theory of mind 


\section{AUTHORS}

\section{NICOLAS CHEVALIER}

Centre for Research in Psychology of Cognition, Language, and Emotion (EA 3273)

University of Provence, 29 Avenue Robert Schuman, 13621 Aix-en-Provence cedex 1, France Nicolas.Chevalier@up.univ-mrs.fr

\section{AGNÈS BLAYE}

Centre for Research in Psychology of Cognition, Language, and Emotion (EA 3273)

University of Provence, 29 Avenue Robert Schuman, 13621 Aix-en-Provence cedex 1, France a.blaye@up.univ-aix.fr 